\title{
Synchronous Ossiculoplasty with Ossicular Replacement Prosthesis during Canal Wall Down Mastoidectomy for Advanced Cholesteatoma: Anatomical and Audiological Outcomes
}

\author{
${ }^{1}$ Mohamed Shareef, ${ }^{2}$ Gul Motwani, ${ }^{3}$ Deepak Verma, ${ }^{4}$ Noor UI Din Malik, ${ }^{5}$ Santosha Ram Choudhary
}

\begin{abstract}
Objectives: To evaluate the anatomical and audiological outcomes of synchronous ossiculoplasty with ossicular replacement prosthesis during canal wall down mastoidectomy for advanced cholesteatoma.

Methods: The prospective study was done at a tertiary referral institute included 30 patients of cholesteatoma who underwent canal wall down mastoidectomy. Ossicular reconstruction was performed using polytetrafluoroethylene (PTFE) (teflon) partial or total ossicular replacement prosthesis. Patients were assessed at 1st, 3rd and 6th postoperative months for graft uptake and hearing evaluation using pure tone audiometry (PTA) in which air bone gap (ABG) closure and mean ABG was calculated and compared with the mean preoperative $A B G$.
\end{abstract}

Results: The mean ABG [both total occicular replacement prosthesis (TORP) and partial occicular replacement prosthesis (PORP) groups combined] considerably reduced from $34.33 \pm$ $4.10 \mathrm{~dB}$ preoperatively to $15.47 \pm 7.65 \mathrm{~dB}$ postoperatively at 6 months. There was a $46.29 \%$ of closure of $A B G$ in 1 st month, which closed more $(53.89 \%)$ in 3rd month and even more $(55.34 \%)$ in 6th postoperative month. $p<0.001$ showed the ABG closure ratio and reduction in the mean $A B G$ was statistically significant in the postoperative period.

Conclusion: Ossicular reconstruction with ossicular replacement prosthesis offers good functional results when performed during canal wall down surgery for advanced cholesteatoma, as a single-stage procedure.

Keywords: Cholesteatoma, Mastoidectomy, Ossiculoplasty, Prosthesis.

How to cite this article: Shareef M, Motwani G, Verma D, Malik NUD, Choudhary SR. Synchronous Ossiculoplasty with Ossicular Replacement Prosthesis during Canal Wall Down Mastoidectomy for Advanced Cholesteatoma: Anatomical and Audiological Outcomes. Int J Otorhinolaryngol Clin 2015;7(3):109-113.

Source of support: Nil

Conflict of interest: None

\footnotetext{
1,3-5 Resident, ${ }^{2}$ Professor

${ }^{1-5}$ Department of ENT, VMMC and Safdarjang Hospital, New Delhi, India
}

Corresponding Author: Deepak Verma, Resident, Department of ENT, VMMC and Safdarjang Hospital, New Delhi, India Phone: 01263210111, e-mail: dpkvrm20@gmail.com

\section{INTRODUCTION}

The surgical management of cholesteatoma has evolved tremendously over ages. The ideal tympanomastoid surgery should eradicate the disease and secondarily reconstruct a sound conduction system to restore the hearing. ${ }^{1}$ This goal can be achieved by a surgical procedure called ossiculoplasty which can be done either as a synchronous (single stage) procedure with the middle ear surgery, or a staged procedure later. In this era of angled endoscopes, visualizing the posterior extent or hidden sites of cholesteatoma like sinus tympani and complete removal of the disease is no longer a difficult task. It remains true that two operations are more costly than one; a second operation places an additional psychological and financial burden upon the patient and the healthcare system. Furthermore, it is difficult to predict what proportion of cases scheduled for a second stage will actually undergo that surgery. Many patients will be satisfied with a dry ear and will not be ready to risk a further procedure. ${ }^{2}$

A wide variety of materials are used in ossiculoplasty like autografts, such as autologous ossicles, cartilage, and bone; homologous grafts, such as homologous bone; and allografts. Allografts have been manufactured in two basic configurations: total ossicular replacement prosthesis (TORP) and partial ossicular replacement prosthesis (PORP). Allografts are made of synthetic materials such as plastipore, hydroxyapatite and titanium. Allografts have an advantage of low extrusion rate, short operative time, risk of residual disease unlikely, and no risk of transmission of disease. ${ }^{3}$

Today, cholesteatoma is not a mortality issue; it is a morbidity problem. Deterioration of quality of life as a result of hearing loss secondary to the disease and sequelae associated with radical operations with extensive mastoidectomy cavities which require lifelong care from moisture and bacteria make these issues a priority. ${ }^{4}$

The purpose of this study is to evaluate the anatomical and audiological outcomes of synchronous ossiculoplasty with ossicular replacement prosthesis during canal wall down mastoidectomy for advanced cholesteatoma. 


\section{METHODS}

Prospective review at a tertiary referral center between 2013 and 2015 included 30 diagnosed patients of cholesteatoma in age group of 12 to 60 years. Patients with severe sensory neural hearing loss/severe mixed hearing loss, malignancy of temporal bone, unresectable cholesteatoma extending down the eustachian tube or into petrous apex, promontory cochlear fistula caused by cholesteatoma were excluded. Ossicular reconstruction was performed using polytetrafluoroethylene (PTFE) (teflon) partial or total ossicular replacement prosthesis, posterior canal wall was reconstructed with either conchal or tragal cartilage in few patients and grafting was done using temporalis fascia. Patients were examined at 1st, 3rd and 6th post operative months for assessment of graft uptake, and hearing evaluation using pure tone audiometry in which $\mathrm{ABG}$ closure and mean $\mathrm{ABG}$ was calculated as mean of frequencies 500, 1000, 2000 and $4000 \mathrm{~Hz}$ and compared with the mean preoperative ABG.

A written informed consent was obtained from all patients before surgery explaining the procedure, possible outcomes and complications. Institutional Ethics Committee clearance was obtained for the study.

\section{Surgical Technique}

All the subjects underwent the said surgical procedure under local/general anesthesia using postaural route. After intraoperative evaluation of the cholesteatoma extent, the decision was made to proceed with canal wall down mastoidectomy in all patients. Disease was completely removed and otoendoscopes were used to ensure the clearance of disease in sinus tympani as and when required. The type of ossiculoplasty was planned according to the status of stapes superstructure. Polytetrafluoroethylene (teflon) PORP with shaft outer diameter $2 \mathrm{~mm}$, internal diameter $1.20 \mathrm{~mm}$ and head diameter $3 \mathrm{~mm}$ while PTFE (teflon) TORP with internal shaft diameter $1 \mathrm{~mm}$ and head diameter $3 \mathrm{~mm}$ was used for ossicular reconstruction. A conchal cartilage graft, along with its perichondrium, was interposed between the prosthesis and the fascial graft (Fig. 1) to provide an extra support to the prosthesis and also helps in the easy conduction of the sound waves from the graft to the stapes. It also ensures that the prosthesis remains in the place and is not extruded in course of time.

The posterior canal wall was reconstructed with either conchal or tragal cartilage in few patients. Adequate meatoplasty was done and cavity was packed with gelfoam and medicated umbilical tape.

\section{RESULTS}

In our study with age group of 12 to 60 years, there were $43.3 \%$ females $(13 / 30)$ and $56.7 \%$ males $(17 / 30)$. The mean ABG (both TORP and PORP groups combined) considerably reduced from $34.33 \pm 4.10 \mathrm{~dB}$ preoperatively to $15.47 \pm 7.65 \mathrm{~dB}$ postoperatively at 6 months (Graph 1 ). There was a $46.29 \%$ of closure of ABG in 1st month, which closed more $(53.89 \%)$ in $3 r d$ month and even more $(55.34 \%)$ in 6th month. $\mathrm{p}<0.001$ showed the ABG closure ratio and reduction in the mean $A B G$ was statistically significant in the postoperative period. Air bone gap of less than $20 \mathrm{~dB}$ was observed in 24 patients ( $80 \%$ ) at the 6 th postoperative month (Graph 2).

All the 30 patients (100\%) had disease involved in epitympanum and mastoid air cells while 16 patients (53\%) had involvement of the mesotympanum, especially the posterior mesotympanum (sinus tympani). In this study, we found that cholesteatoma localization had no statistically significant effect on postoperative ABG (Table 1).

The Austin classification was used to define each patient's ossicular status and depending on which patients were classified (Table 2).

Partial ossicular replacement prosthesis was used in 6 patients while 24 patients underwent ossicular reconstruction using TORP. Graph 3 shows correlation of audiological success rate with the type of prosthesis used.
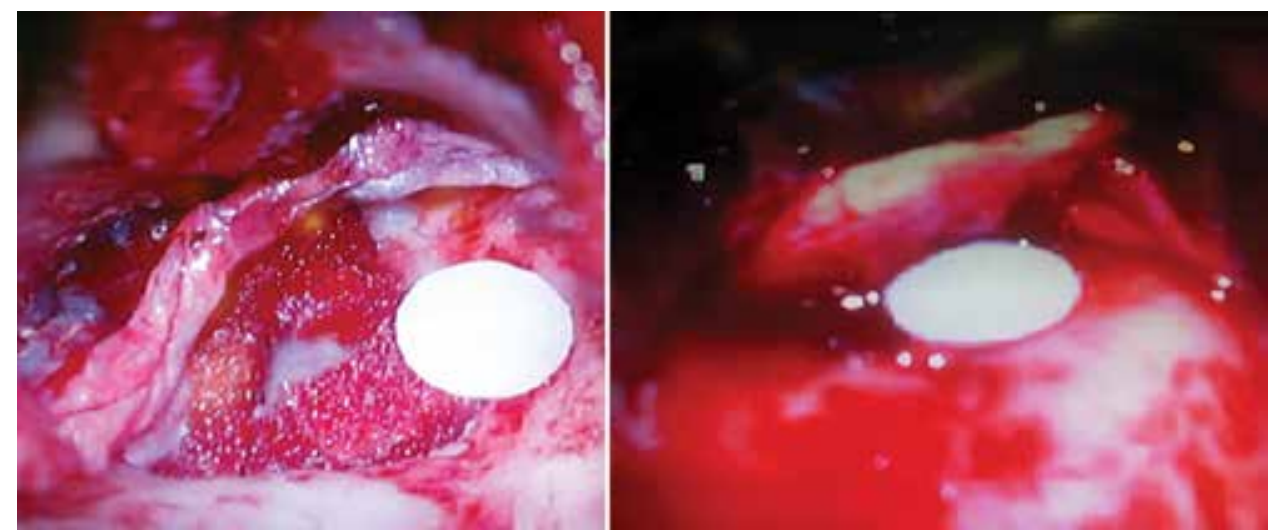

Fig. 1: A photograph showing prosthesis supported by gelfoam in middle ear and cartilage placed between the prosthesis and graft 


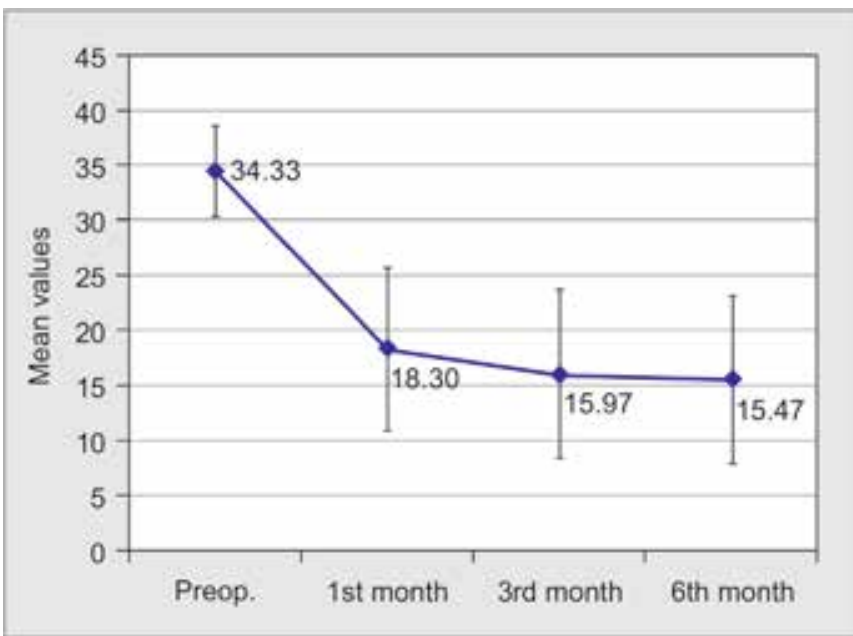

Graph 1: Distribution of mean $A B G$ over a period of 6 months

Table 1: Correlation of audiological success rate in relation to extent of cholesteatoma

\begin{tabular}{|c|c|c|c|c|}
\hline \multirow[b]{2}{*}{$\begin{array}{l}\text { Extent of } \\
\text { cholesteatoma }\end{array}$} & \multirow[b]{2}{*}{ Total } & \multicolumn{3}{|c|}{$A B G<20 d B$} \\
\hline & & $\begin{array}{l}1 s t \\
\text { month }\end{array}$ & $\begin{array}{l}\text { 3rd } \\
\text { month }\end{array}$ & $\begin{array}{l}6 \text { th } \\
\text { month }\end{array}$ \\
\hline $\begin{array}{l}\text { Epitympanum + } \\
\text { mastoid antrum } \\
\text { and air cells }\end{array}$ & 30 & $\begin{array}{l}19 \\
(63.3 \%)\end{array}$ & $\begin{array}{l}23 \\
(76.7 \%)\end{array}$ & $\begin{array}{l}24 \\
(80 \%)\end{array}$ \\
\hline $\begin{array}{l}\text { Epitympanum + } \\
\text { mastoid antrum } \\
\text { and air cells + } \\
\text { mesotympanum }\end{array}$ & 16 & $\begin{array}{l}8 \\
(50 \%)\end{array}$ & $\begin{array}{l}11 \\
(68.8 \%)\end{array}$ & $\begin{array}{l}11 \\
(68.8 \%)\end{array}$ \\
\hline
\end{tabular}

There were 2 patients of lateral semicircular canal dehiscence, whereas 1 patient had dehiscence of fallopian canal and sigmoid plate. Graft failure occurred in 3 patients. The prosthesis and the cartilage over the prosthesis were also extruded in all the three patients leading to postoperative ear discharge and residual hearing loss. Though one of the patient, failed to follow-up after 6 months, the rest 2 patients were taken up for second stage tympanoplasty later. One patient had granulation in the postoperative cavity while no patient had recurrence of cholesteatoma.

\section{DISCUSSION}

Single stage canal wall down mastoidectomy with ossiculoplasty in the same sitting using ossicular replacement prosthesis is an effective and successful mode of surgical management of cholesteatoma and restoration of the hearing mechanism.

Extent of cholesteatoma in different areas of the middle ear like epitympanum including anterior epitympanic sinus, mesotympanum including posterior mesotympanum (sinus tympani), extending medial to ossicles, mastoid air cells and antrum is an important prognostic factor for the audiological and anatomical

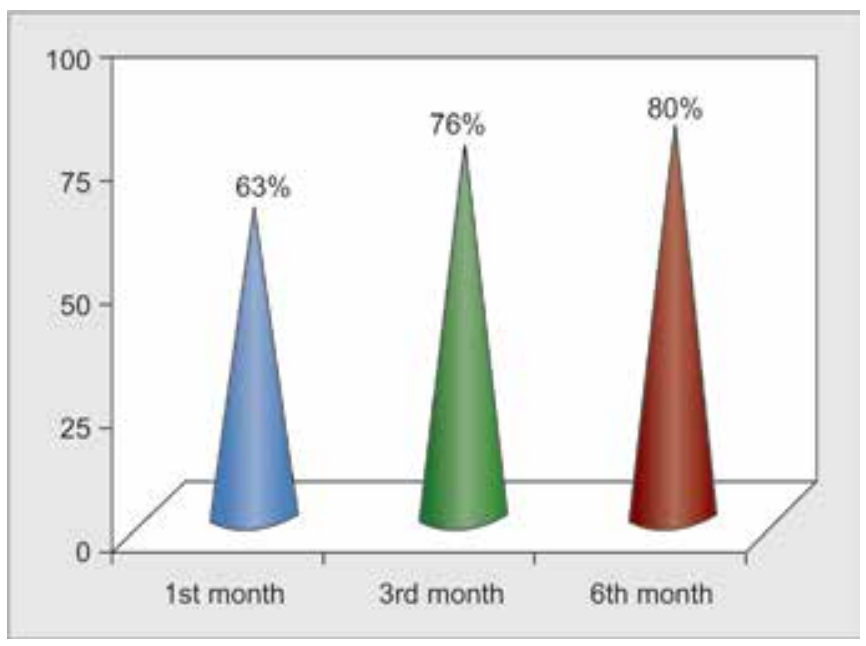

Graph 2: Audiological success rate of the surgery $(A B G<20 \mathrm{~dB})$ in $1 \mathrm{st}, 3 \mathrm{rd}$ and 6 th postoperative period

Table 2: Correlation of Austin's classification and audiological success rate of surgery

\begin{tabular}{|c|c|c|c|c|}
\hline \multirow[b]{2}{*}{$\begin{array}{l}\text { Austin } \\
\text { classification }\end{array}$} & \multirow[b]{2}{*}{$\begin{array}{l}\text { No. of } \\
\text { patients }\end{array}$} & \multicolumn{3}{|c|}{$A B G<20 d B$} \\
\hline & & $\begin{array}{l}\text { 1st } \\
\text { month }\end{array}$ & $\begin{array}{l}\text { 3rd } \\
\text { month }\end{array}$ & $\begin{array}{l}\text { 6th } \\
\text { month }\end{array}$ \\
\hline $\mathrm{M}-\mathrm{S}-$ & 6 & $\begin{array}{l}1 \\
(16.7 \%)\end{array}$ & $\begin{array}{l}2 \\
(33.3 \%)\end{array}$ & $\begin{array}{l}2 \\
(33.3 \%)\end{array}$ \\
\hline $\mathrm{M}-\mathrm{S}+$ & 1 & $\begin{array}{l}0 \\
(0 \%)\end{array}$ & $\begin{array}{l}0 \\
(0 \%)\end{array}$ & $\begin{array}{l}1 \\
(100 \%)\end{array}$ \\
\hline$M+S-$ & 18 & $\begin{array}{l}14 \\
(77.8 \%)\end{array}$ & $\begin{array}{l}17 \\
(94.4 \%)\end{array}$ & $\begin{array}{l}17 \\
(94.4 \%)\end{array}$ \\
\hline $\mathrm{M}+\mathrm{S}+$ & 5 & $4(80 \%)$ & $4(80 \%)$ & $4(80 \%)$ \\
\hline$p$-value & & $0.023^{*}$ & $0.005^{\star}$ & $0.013^{*}$ \\
\hline
\end{tabular}

*Statistically significant

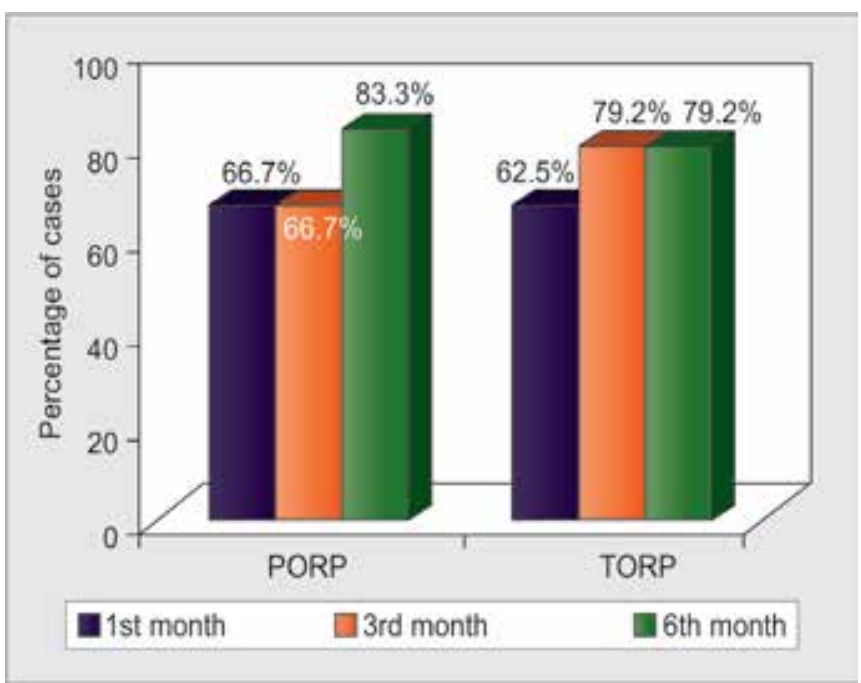

Graph 3: Correlation of audiological success rate and the type of prosthesis used [success rate $(A B G<20)$ in TORP and PORP]

success of the surgery. The most important factor during surgical management of attic cholesteatomas is preservation of the middle-ear cavity, in order to improve the functional result. Thus, one may think of better results for patients with epitympanic cholesteatoma, 
because of the greater possibility of middle-ear cavity preservation. $^{2}$ However, in our study and the other conducted by Iseri et al, the cholesteatoma location did not affect patient's hearing outcomes in terms of postoperative ABG. The extension of cholesteatoma into the posterior mesotympanum had a striking effect on the stapes superstructure and can partially or totally erode the ossicle by its enzymatic action and thus became a factor determining the type of tympanoplasty post canal wall down mastoidectomy. Some authors have reported that the loss of the stapes suprastructure is associated with a significantly poorer auditory outcome. ${ }^{5}$ We found similar results in our study. Sixteen out of the 30 patients had disease involving the sinus tympani area and out of these 16 only one patient $(6.3 \%)$ had an intact stapes superstructure, rest 15 had only a mobile footplate of stapes.

In our study, the mean ABG (both TORP and PORP groups combined) considerably reduced from $34.33 \pm 4.10 \mathrm{~dB}$ preoperatively to $15.47 \pm 7.65 \mathrm{~dB}$ postoperatively at 6 months which is statistically significant and comparable with the results obtained by Iseri et al, where the mean ABG improved from $30.38 \pm 11.12 \mathrm{~dB}$ preoperatively to $15.62 \pm 9.65 \mathrm{~dB}$ postoperatively, for both groups combined. ${ }^{2}$ Similarly, Begall and Zimmermann reported a $15 \mathrm{~dB}$ ABG improvement at 6 months' follow-up; while Schmerber et al reported a $12.7 \mathrm{~dB}$ ABG improvement at 20 months' follow-up. ${ }^{6,7}$

The meatoplasty is often a neglected but very important part of mastoidectomy operation as it helps to assess the patient's postoperative cavity for presence of any discharge, crusts or any residual or recurrent cholesteatoma. One patient, in whom, the meatoplasty was not adequate and who had granulation in the cavity continue to have ear discharge even at 6th month. This underlines the significance of an adequate meatoplasty in canal wall down surgery.

Reconstruction of the posterior canal wall helps to maintain an anatomically normal canal, prevents the postoperative cavity problems like discharge, debris and increases the patient compliance as the patient need not come to hospital repeatedly to clean the postoperative cavity. Eleven out of 30 patients (36.7\%) had the posterior canal wall reconstruction done with either tragal or conchal cartilage (Fig. 2).

\section{CONCLUSION}

Ossicular reconstruction with ossicular replacement prosthesis offers good functional results when performed during canal wall down surgery for advanced cholesteatoma, as a single-stage procedure. This procedure

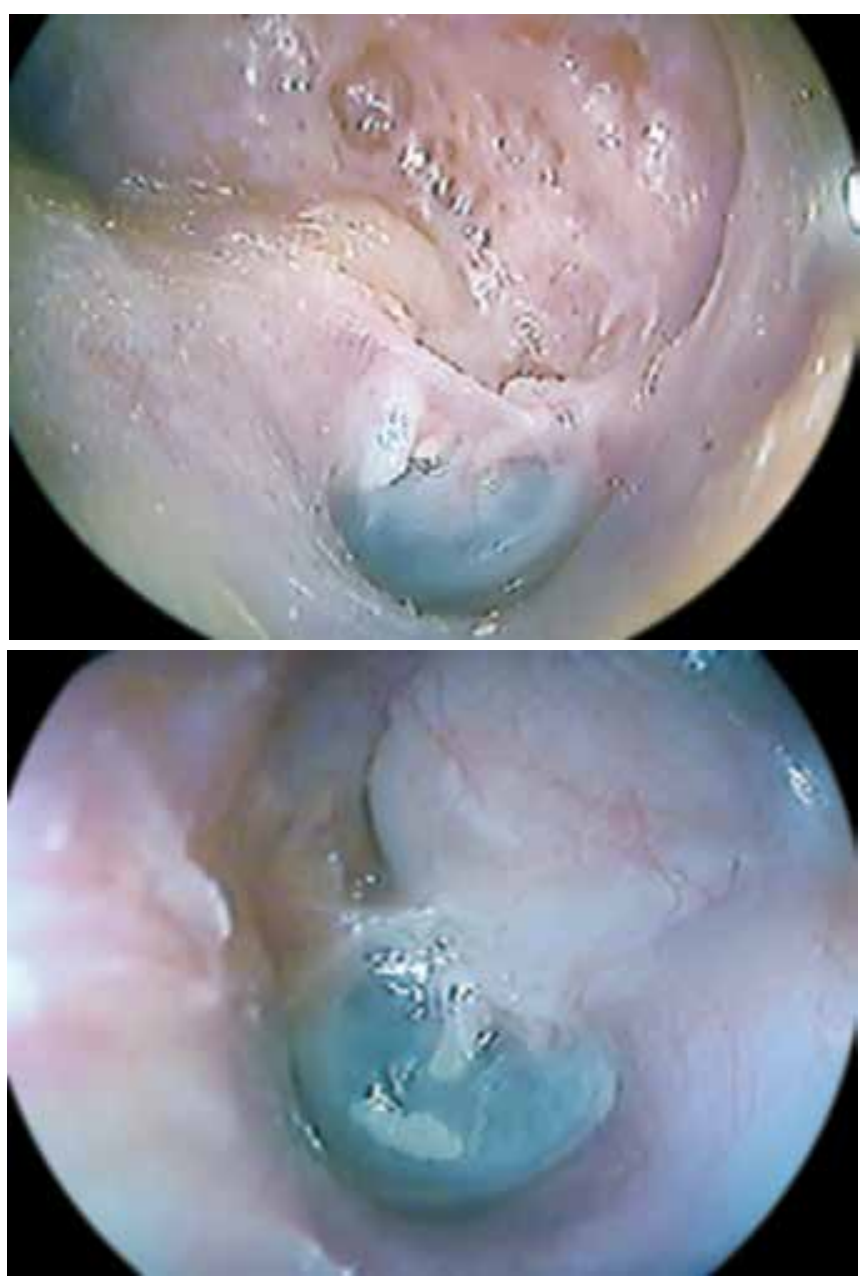

Fig. 2: A photograph showing well epithelized postoperative cavity at 6th month with healthy graft and after posterior canal wall reconstruction

benefits the patient by avoiding a second surgery and thus decreases psychological and financial burden upon the patient and the healthcare system.

Audiological success of the surgery significantly dependents on several factors like Austin's classification, the presence or absence of the stapes superstructure, the type of prosthesis used and the type of tympanoplasty done.

\section{REFERENCES}

1. Demir UL, Karaca S, Ozmen OA, Kasapoglu F, Coskun HH, Basut $\mathrm{O}$. Is it the middle ear disease or the reconstruction material that determines the functional outcome in ossicular chain reconstruction? Otol Neurotol 2012;33(4): 580-585.

2. Iseri M, Ustundaq E, Ulubil A, Ozturk M, Bircan O. Synchronous ossiculoplasty with titanium prosthesis during canal wall down surgery for advanced cholesteatoma: anatomical and hearing outcomes. J Laryngol Otol 2012;126(2):131-135.

3. De Corso E, Marchese MR, Sergi B, Rigante M, Paludetti G. Role of ossiculoplasty in canal wall down tympanoplasty for 
middle-ear cholesteatoma: hearing results. J Laryngol Otol 2007;121(4):324-328.

4. Blanco P, Gonzalez F, Holgun J, Guerra C. Surgical management of middle ear cholesteatoma and reconstruction at the same time. Colomb Med 2014;45(3):127-131.

5. Alaani A, Raut VV. Kurz titanium prosthesis ossiculoplastyfollow-up statistical analysis of factors affecting 1 year hearing results. Auris Nasus Larynx 2010;37(2):150-154.
6. Begall $\mathrm{K}$, Zimmermann $\mathrm{H}$. Reconstruction of the ossicular chain with titanium implants: results of a multicenter study. Laryngorhinootologie 2000;79(3):139-145.

7. Schmerber S, Troussier J, Dumas G, Lavieille JP, Nguyen D. Hearing results with the titanium ossicular replacement prostheses. Eur Arch Otorhinolaryngol 2006;263(4): 347-354. 